\title{
Téoros
}

Revue de recherche en tourisme

\section{Pour une intégration du service à la clientèle au marketing hôtelier}

\section{Jocelyn D. Perreault et Denis Pettigrew}

Volume 23, numéro 3, automne 2004

Regards sur l'hôtellerie : un secteur en mutation

URI : https://id.erudit.org/iderudit/1071231ar

DOI : https://doi.org/10.7202/1071231ar

Aller au sommaire du numéro

Éditeur(s)

Université du Québec à Montréal

ISSN

0712-8657 (imprimé)

1923-2705 (numérique)

Découvrir la revue

Citer cet article

Perreault, J. D. \& Pettigrew, D. (2004). Pour une intégration du service à la clientèle au marketing hôtelier. Téoros, 23(3), 37-41.

https://doi.org/10.7202/1071231ar d'utilisation que vous pouvez consulter en ligne.

https://apropos.erudit.org/fr/usagers/politique-dutilisation/ 


\section{Pour une intégration du service à la clientèle au marketing hôtelier}

\section{Jocelyn D. Perreaulf et Denis Pettigrew}

Cet article veut proposer une vision intégrée du service à la clientèle et de la planification stratégique en marketing, démarche qui s'avère cruciale dans le domaine de la gestion hôtelière. Nous proposons d'abord un modèle représentant le processus de planification stratégique en marketing (Pettigrew et Turgeon, 2004) est d'abord proposé, suivi d'une schématisation des spécificités du marketing des services et du processus de servuction (Eiglier et Langeard, 1987). Nous présentons en- suite certaines définitions du service à la clientèle, dont celle que nous privilégions. Nous précisons également comment le service à la clientèle s'intègre aux divers concepts retenus et au processus décisionnel du consommateur de services hôteliers. En guise de conclusion, nous proposons des pistes de recherches futures.

Comme on le constate à la figure 1, le processus de planification stratégique débute avec la collecte d'information (données secondaires) sur l'environnement externe. Il s'agit, en fait, de prendre la photo la plus claire et la plus exhaustive possible afin de bien identifier les règles du jeu qui y pré- valent et, surtout, de bien anticiper les tendances qui s'y dessinent. On doit pouvoir anticiper à quoi ressemblera la photo dans six mois, dans un an, etc. Il est impératif de bien comprendre et de prévoir le plus précisément possible le rôle et l'importance des divers facteurs (économiques, technologiques, par exemple) et des divers acteurs en cause (concurrents, organismes de réglementation, par exemple). La collecte de données primaires auprès des divers acteurs vient valider, compléter ou bonifier les données secondaires préalablement obtenues. Cette première étape correspond au diagnostic externe pour une entreprise de services hôteliers.

\section{FIGURE 1 : Le processus de planification stratégique en marketing}

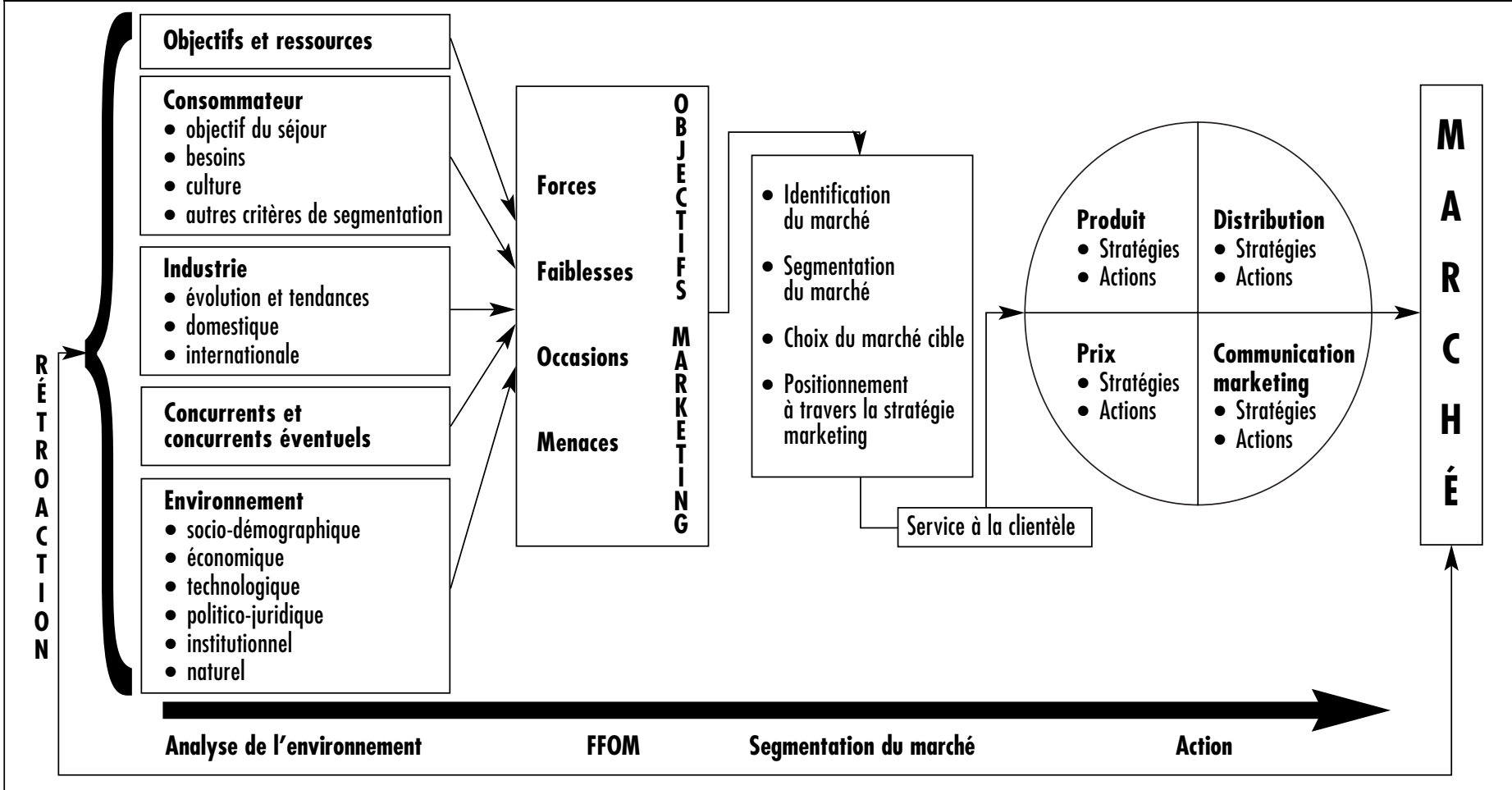

Source: Adapté de Pettigrew et Turgeon, 2004 : 73. 


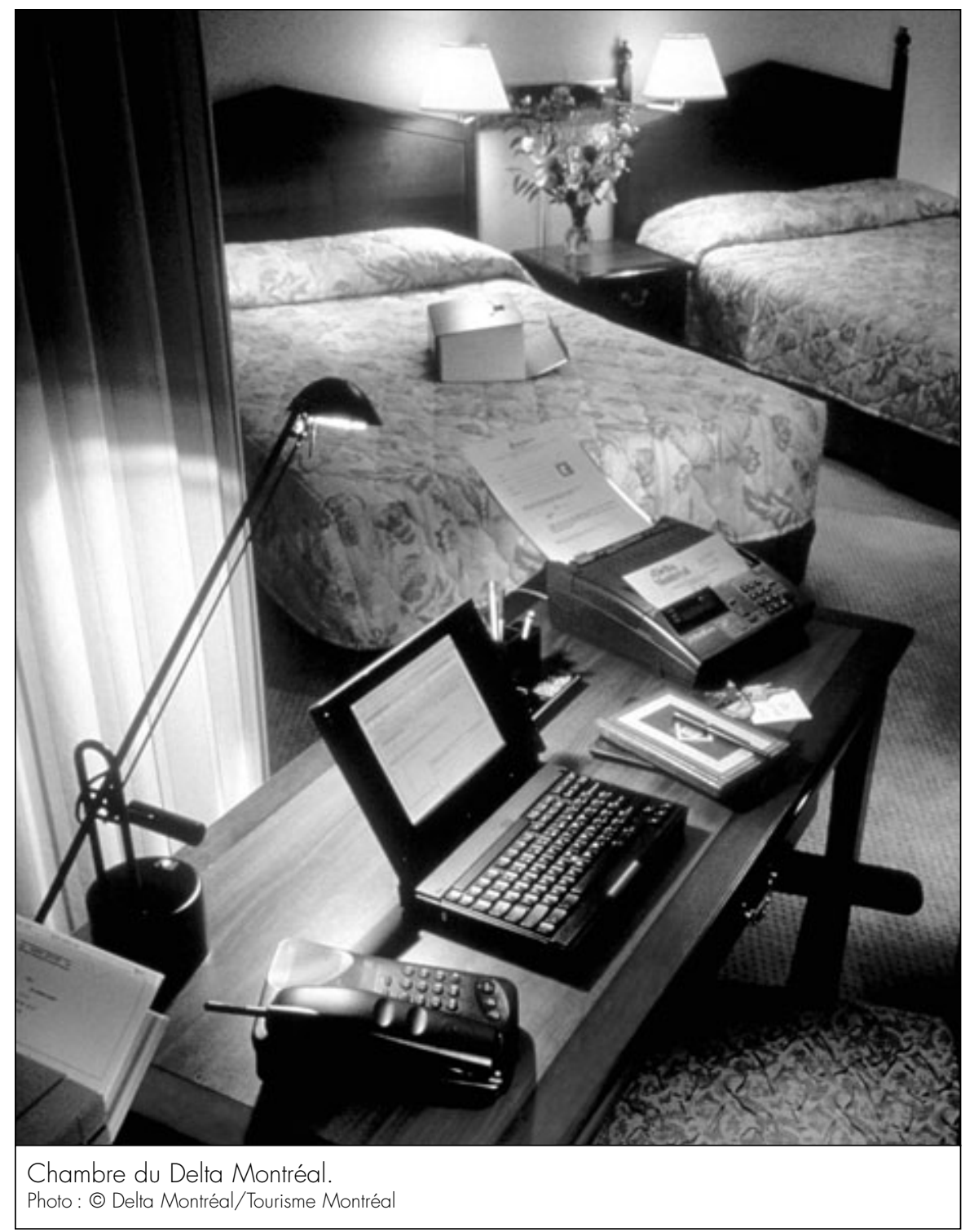

FIGURE 2: La servuction de l'entreprise de service

Entreprise de service

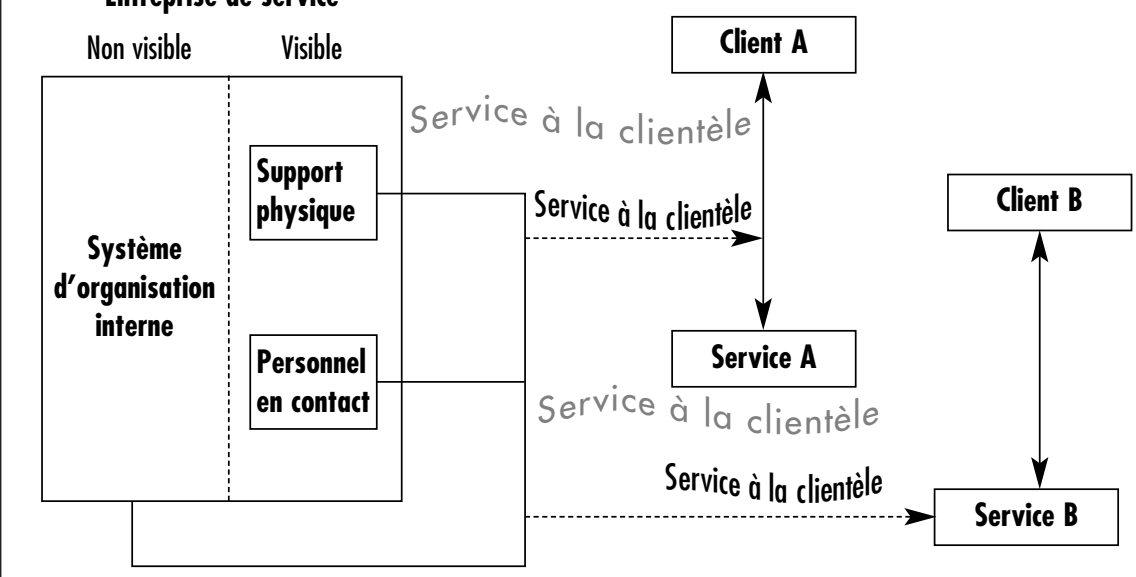

Source : Adapté de Eiglier et Langeard, 1987: 18
La deuxième étape consiste à procéder au diagnostic interne, c'est-à-dire à identifier clairement les forces et les faiblesses de l'organisation et à faire l'inventaire de toutes ses ressources (humaines, financières, connaissances, etc.). La confrontation des deux diagnostics permettra, par la suite, d'identifier les menaces et les occasions qui découlent des deux réalités interne et externe et forcera le gestionnaire marketing à prendre les décisions stratégiques et opérationnelles requises. La première décision stratégique est le choix d'un segment ou d'un marché spécifique auquel l'organisation sera appelée à offrir ses services, cette offre de services étant la deuxième décision stratégique. Ces deux grandes décisions stratégiques doivent être en conformité avec le positionnement retenu ou choisi suivant l'analyse préalable des occasions et des menaces.

Le service à la clientèle apparaît dans le modèle au niveau décisions stratégiques, car il devient par le fait même un élément de positionnement indéniable qui influencera toutes les décisions subséquentes qui devront être prises par les responsables du marketing. Le service à la clientèle apparaît également comme un élément central pour Samii (2001), alors qu'il découle de l'analyse marketing et qu'il oriente l'ensemble des décisions de la distribution et de la logistique. Nous y reviendrons plus en détail subséquemment.

\section{La servuction ou la production de services}

Il est impossible d'aborder le thème de la gestion hôtelière sans aborder celui des services. Eiglier et Langeard (1987) ont proposé un néologisme pour l'étude du marketing des services : la servuction. Il s'agit d'un terme pour désigner le processus de production des services, lequel s'applique à notre cas du marketing hôtelier. Le modèle proposé par ces auteurs (voir figure 2) comporte trois grandes composantes: l'entreprise de service, les clients, les interactions clients-entreprises et clients-clients. L'entreprise de service se compose d'une partie non visible ou de l'organisation interne, laquelle n'est pas en contact direct avec les clients. La partie visible, quant à elle, comprend les composantes physiques de l'entreprise (édifice, ameublement, équipement informatique, etc.) et le personnel qui entre généralement en contact avec la clientèle. On note que le service à la clientèle est l'affaire de tous les acteurs de l'entreprise, autant dans l'organisation interne que le personnel en contact. 
La figure 3 met en évidence les divers types de services compris dans l'offre faite par l'entreprise. On y note le service de base principal (SBP) qui comprend l'ensemble de tous les services périphériques (P1 à P6) offerts et disponibles à la clientèle. L'objectif ultime est que chaque service périphérique permette à l'entreprise de service de se différencier et lui procure un avantage concurrentiel. Dans le secteur hôtelier, par exemple, le service de base ou la raison d'être est l'hébergement, alors que les services périphériques pourraient comprendre les salles de réception, la piscine, le sauna, la salle d'entraînement, etc. Le service de base dérivé (SBD) correspond à un ou à des services considérés autonomes, s'adressant à des clientèles particulières, mais tout de même disponibles à la clientèle de base. Il peut s'agir, par exemple, d'un centre de thalassothérapie de grande notoriété.

\section{Le service à la clientèle : certaines définitions}

Comme le notait déjà Peel (1990), toutes les organisations, qu'elles soient du secteur public ou privé, reconnaissent l'importance du service à la clientèle, bien que sa définition soit trop fréquemment restrictive (Boehr, 2004). Pour Peel, un bon service à la clientèle débute même avant que la transaction ait lieu, et comprend le climat qui entoure l'étude de marché, le processus d'achat et de commande, la période d'attente jusqu'à la livraison s'il y a lieu, l'emballage et la présentation du produit, le transport et la logistique, la gestion des plaintes, le paiement et les mécanismes de récupération des comptes en souffrance et tout l'ensemble des services après-vente. On constate que, pour cet auteur, l'horizon du service à la clientèle est très élargi.

Selon Boehr (2004), le service à la clientèle est une notion qui, à l'heure actuelle, dépasse la simple et vague idée du service après-vente. Bien plus que cela, il s'agit d'une application qui relève du secteur tertiaire et qui s'avère être présente dans toutes les entreprises. Toute société, qu'il s'agisse d'une entreprise individuelle, d'une PME ou d'une entreprise multinationale, pratique le service à la clientèle.

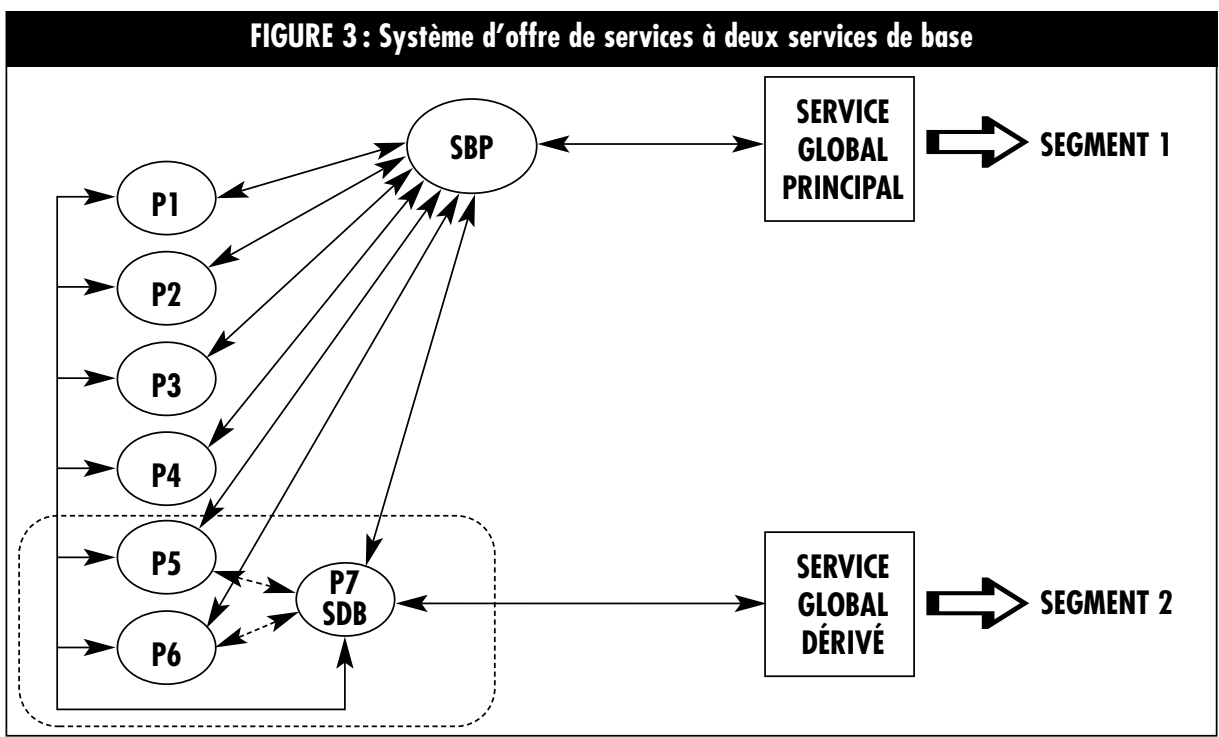

Source: Eiglier et Langeard, 1987: 87.

Pour Boehr (2004), il n'existerait donc pas une seule organisation qui propose ou pas un service à la clientèle, lequel ne réside ni dans la fabrication ou l'achat d'un produit pour le vendre ou le revendre, ni dans le fait de proposer des services, comme c'est le cas dans l'industrie des services telle que nous la connaissons. Bien au contraire, cette application que l'on trouve dans toutes les entreprises consiste à accompagner, à suivre ou encore à guider le ou les prospects ou clients, dès l'entrée d'un site Internet, d'un magasin, d'un magazine, d'une brochure, d'un dépliant ou de tout autre support informationnel, afin de les mettre dans de bonnes dispositions et ce, jusqu'à la sortie, en créant ainsi, chez le client ou le prospect, l'envie d'achat ou de retour sur ce même vecteur d'information. Le but, selon cet auteur, est de prendre en charge le client, de satisfaire ses besoins, ses attentes et de faire en sorte, soit qu'il ne sorte pas les mains vides, soit qu'il revienne, soit qu'il développe, par le bouche à oreille, l'image de marque ainsi que la renommée de l'entreprise. Car la finalité d'un service à la clientèle, c'est, d'une part, de satisfaire le client et, d'autre part, d'attirer de nouveaux prospects et, finalement, de les fidéliser.

Coupet (1990) maintient que le service à la clientèle signifie avant tout une relation, une relation humaine de qualité. Il s'agirait d'un pivot autour duquel les entreprises déploient leurs ressources. Cet auteur le voit même comme une nouvelle fonction au confluent du marketing et de la gestion des ressources humaines. En définitive, selon Coupet (1990), la qualité de la gestion des ressources humaines et les gestionnaires du service à la clientèle n'ont pas d'autre choix que de se définir comme des agents de changement. Macaulay et Cook (1993) maintiennent que le service à la clientèle est la clé du succès pour une organisation et que ce dernier consiste à:

- faire ce que l'on a dit que l'on ferait ;

- faire sa priorité de ce qui compte pour le consommateur;

- trouver des façons de s'améliorer;

- développer des contacts personnels positifs avec le consommateur; et

- compter sur du personnel bien entraîné, motivé, qui travaille bien ensemble.

Pour Gemme (2004), le service à la clientèle se définit principalement par la relation qui existe entre l'entreprise et les clients. Dans le même sens, gérer le service à la clientèle consiste, selon Désormeaux et Lafrance (2001), à gérer l'ensemble des expériences ainsi que les attentes des clients, de façon à satisfaire le mieux possible la clientèle cible de l'entreprise.

Boehr (2004) a vérifié, au début de l'été 2004, comment les propriétaires-dirigeants des gîtes du passant de la région trifluvienne définissaient le service à la clientèle. Les questions posées étaient les suivantes: 
«Selon vous, à quoi correspond le service à la clientèle? Comment le percevez-vous? Si vous aviez une définition à me donner, quelle serait-elle?»

Quatre visions ou définitions ont été mises de l'avant :

1. Le service à la clientèle, c'est mettre à la disposition des clients une infrastructure propre, accueillante, confortable.

2. Le service à la clientèle, c'est agir comme si l'on recevait des amis ; c'est la convivialité.

3. Le service à la clientèle, c'est la satisfaction du client ; c'est le domaine du plaisir, de la jouissance et que le tout soit agréable.

4. Le service à la clientèle consiste à être le plus près possible du client, à créer une atmosphère.

On note ici que l'aspect relationnel est omniprésent dans la définition que se font les propriétaires-dirigeants des gîtes (Bed and Breakfast) rencontrés.

\section{Notre définition du service à la clientèle}

La définition que nous retenons, pour les fins de cet article, est celle déjà proposée par Perreault (2004) dans un document de travail du Laboratoire de recherche et d'intervention en gestion hôtelière et restauration, à l'effet que le service à la clientèle est: «L'art d'accompagner le client à partir de l'identification de ses besoins jusqu'à sa fidélisation. »

Le mot art pour :

- (A) Activités devant être réalisées dans le cadre de la stratégie de service à la clientèle = ce que l'on fait ou devrait faire.

- (R) Renouvellement de la façon de le faire; se différencier de l'ensemble de l'industrie; se procurer un avantage concurrentiel, innover dans les activités, les méthodes, etc. $=$ ce que l'on change.

Il s'agit donc ici de «faire» du changement.

- (T) Transformation de l'organisation, de la façon d'être; de la structure et du rôle des divers acteurs impliqués $=$ ce que l'on devient.

Il s'agit donc ici de la gestion du changement.
Selon la définition que nous retenons, le service à la clientèle intervient au niveau pré-transactionnel (communiquer de l'information) ; aux niveaux transactionnel (gérer l'interaction) et post-transactionnel (établir et maintenir une relation). Ces trois niveaux se situent tout au long du processus décisionnel du consommateur qui suit :

- Identification des besoins

- Recherche d'information interne et externe

- Se compromettre en visitant un site Web ou l'entreprise

- Création d'une attitude favorable, d'une préférence

- Choix

- Consommation

- Satisfaction

- Fidélisation

Il s'agit donc d'accompagner le client jusqu'au maintien d'une relation stable et durable, soit sa fidélisation, situation à laquelle le client devient lui-même un agent de changement en proposant des activités, des changements, une nouvelle façon d'être, un nouvel ART du service à la clientèle.

S'inspirant des travaux de Bégin et Boisvert (2002), Ouedrango (2004) a évalué cinquante sites Web d'entreprises hôtelières afin de voir s'ils contribuaient à un meilleur service à la clientèle pour les clients de ces organisations. À partir de quatorze critères d'évaluation, Ouedrango (2004) conclut que les sites Web contribuent significativement aux toutes pre- mières étapes du processus de consommation des services. C'est-à-dire que, dans l'ensemble, ils contribuent tous, à divers degrés, à un accompagnement amélioré du consommateur dans le choix d'un établissement hôtelier.

\section{La satisfaction des consommateurs à l'égard des services obtenus}

Un partenariat établi entre la University of Michigan Business School, l'American Society for Quality et une firme internationale de consultants, le CFI Group, a permis de proposer un indice qui reflète les tendances de satisfaction des consommateurs américains. Cet indice fournit des renseignements de benchmarking pour les entreprises, les associations d'affaires et les agences gouvernementales. Le tableau 1 met en évidence un portrait global de la satisfaction des consommateurs à partir de la date de référence ou de départ de l'indice jusqu'à 1999.

La compilation des résultats de l'ACSI pour la période de référence 1994-1999 démontre une baisse de la satisfaction des consommateurs variant de $1 \%$ à $4 \%$ dans l'ensemble des secteurs de l'économie américaine. Seuls les services publics américains ont démontré une très légère hausse. Les secteurs les plus touchés sont ceux des transports, des communications, des services d'utilités, des services d'hébergement, de la restauration et de la santé. La baisse observée pour cette période est d'environ $4 \%$. D'ailleurs des signes d'inefficacité se fai-

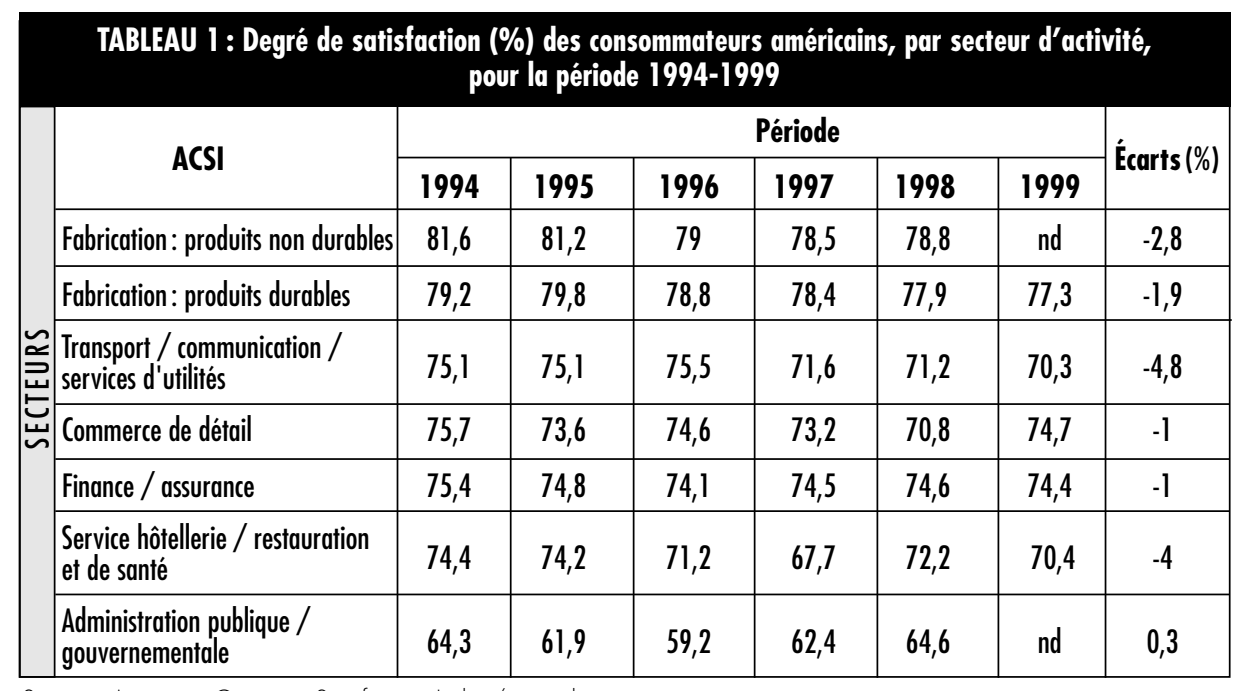

Source: American Customer Satisfaction Index/www.theasci.org 
TABLEAU 2: Observations récentes de l'évolution de l'indice ACSI pour le secteur hôtelier (1994-2004)

\begin{tabular}{|l|c|c|c|c|c|c|c|c|}
\hline & 1994 & $\mathbf{2 0 0 0}$ & $\mathbf{2 0 0 1}$ & $\mathbf{2 0 0 2}$ & $\mathbf{2 0 0 3}$ & $\mathbf{2 0 0 4}$ & $\begin{array}{c}\text { Chang. } \\
2003-2004\end{array}$ & $\begin{array}{c}\text { Chang. } \\
1994-2004\end{array}$ \\
\hline SERVICES & 74,4 & 69,4 & 68,8 & $\mathrm{NM}$ & 72,8 & 74,7 & $2,6 \%$ & $0,4 \%$ \\
\hline HOTEIS & 75 & 72 & 71 & 71 & 73 & 72 & $-1,4 \%$ & $-4,0 \%$ \\
\hline Hilton Hotels Corporation & 75 & 77 & 74 & 76 & 74 & 77 & $4,1 \%$ & $2,7 \%$ \\
\hline Marriott International, Inc. & 80 & 74 & 77 & 76 & 76 & 76 & $0,0 \%$ & $-5,0 \%$ \\
\hline Hyatt Corporation & 76 & 74 & 73 & 75 & 77 & 74 & $-3,9 \%$ & $-2,6 \%$ \\
\hline $\begin{array}{l}\text { Holiday Inn (InterConti- } \\
\text { nental Hotels Group PLC) }\end{array}$ & 69 & 71 & 71 & 69 & 72 & 73 & $1,4 \%$ & $5,8 \%$ \\
\hline $\begin{array}{l}\text { Starwood Hotels \& Resorts } \\
\text { Worldwide, Inc. }\end{array}$ & $\mathrm{NM}$ & 73 & 71 & 69 & 73 & 73 & $0,0 \%$ & $0,0 \%$ \\
\hline All Others & $\mathrm{NM}$ & 72 & 70 & 70 & 72 & 71 & $-1,4 \%$ & $-2,7 \%$ \\
\hline $\begin{array}{l}\text { Ramada Franchise Systems Inc. } \\
\text { (Cendant Corporation) }\end{array}$ & 70 & 69 & 66 & 67 & 70 & 67 & $-4,3 \%$ & $-4,3 \%$ \\
\hline HOSPITALS & 74 & 69 & 68 & 70 & 73 & 76 & $4,1 \%$ & $2,7 \%$ \\
\hline MOTION PICTURES & 77 & 68 & 71 & 70 & 71 & 73 & $2,8 \%$ & $-5,2 \%$ \\
\hline
\end{tabular}

Source : American Customer Satisfaction Index/www.theasci.org

saient déjà sentir durant la période 1985-1990, en particulier dans le secteur des services du transport aérien, des institutions financières et du commerce de détail.

De manière générale, pour la période de référence, l'efficacité des services s'est améliorée au début des années 1994, pour ensuite se détériorer graduellement jusqu'en 1999, dernière année d'observation de ce tableau. Cette période choc correspond au grand bouleversement causé par la révolution de l'information et par la nécessité qu'ont eu les entreprises d'utiliser rapidement des stratégies d'adaptation, en particulier de fusion / acquisition afin de retrouver leur rentabilité face à une concurrence féroce dans un marché morose et offrant peu d'occasions.

Le tableau 2 met plus précisément l'accent sur la période 1994-2004 et sur le secteur des services, soit l'hôtellerie et la restauration, la santé et le divertissement (cinématographique), en comparant l'indice de référence de 1994 au premier trimestre de 2004. On observe dans l'ensemble que le degré de satisfaction des consommateurs s'est maintenu pour le secteur des services, mais s'est détérioré dans les dix dernières années pour le secteur hôtelier $(-4,0 \%)$ et l'industrie cinématographique $(-5,2 \%)$, alors qu'il s'est amélioré de $2,7 \%$ pour les établissements de santé. À noter (tableau 1) qu'il existe un écart important entre le taux de satisfaction obtenu dans le secteur hôtelier par rapport aux autres secteurs plus performants, d'autant plus que cela intervient à un moment critique du processus de décision du consommateur.

\section{Conclusion}

Les données récentes de l'indice ACSI semblent indiquer que le secteur hôtelier aurait tout un chemin à parcourir afin d'augmenter le niveau de satisfaction de sa clientèle. Aucune donnée ne nous laisse croire que l'industrie canadienne n'a pas le même défi à relever, surtout que le profil des entreprises du secteur hôtelier canadien est principalement formé de PME; imaginer alors le niveau de satisfaction obtenu par les entreprises qui disposent de ressources limitées. Il nous semble donc que l'amélioration du service à la clientèle soit une piste intéressante à explorer dans ce sens.

\section{Jocelyn D. Perreault et Denis Petti-} grew sont D Sc. du Département des sciences de la gestion de l'Université du Québec à Trois-Rivières et membres du Laboratoire de recherche et d'intervention en gestion hôtelière et restauration.

\section{Bibliographie}

ACSI (American Customer Satisfaction Index) (2004), ACSI scores for Q1 2004,

[http://www.theacsi.org].

Albrecht, K., et R. Zemke (1985), Service America, Dow Jones Irwin.

Bégin, L., et H. Boisvert (2002), Rentabiliser le commerce électronique, quel rôle joue Internet dans votre stratégie d'entreprise, CMA Management, décembre-janvier, vol. 75, $\mathrm{n}^{\circ}$ 9, p. 25-27.

Boehr, J.-L. (2004), Évaluation de la qualité du service à la clientèle, document de travail inédit, Département des sciences de la gestion, Université du Québec à Trois-Rivières, printemps-été. Colbert et al. (2002), Gestion du Marketing, Gaétan Morin éditeur, $3^{\mathrm{e}}$ édition, 460 p.

Coupet, A. (1990), «Le service à la clientèle : De la stratégie de marketing à la gestion de la qualité », Revue Gestion, novembre, vol. 15, no 4, p. 27-36.

Désormeaux, Robert, et Serge Lafrance (2001), «Le mythe de l'infidélité des consommateurs », Revue Gestion, vol. 26, n³ 3, p. 98-103.

Eiglier, P., et Éric Langeard (1987), Servuction : Le marketing des services, Collection Stratégie et management, McGraw-Hill.

Gemme, Mélissa (2004), Évaluation de la satisfaction à l'égard du service à la clientèle: Le cas d'une PME de services québécoise, mémoire de maîtrise en gestion des PME, Université du Québec à Trois-Rivières.

Macaulay, S., et S. Cook (1993), «Seven Essentials for Managing Superior Customer Service », Training for quality, vol. 1, $\mathrm{n}^{\circ} 2$, Emerald Group Publishing Limited.

Ouedrango (2004), Contribution des sites Web hôteliers au processus décisionnel des consommateurs, projet d'application en administration, réalisé au printemps 2004, Département des sciences de la gestion, Université du Québec à Trois-Rivières.

Peel, Malcolm (1990), "Customer Service», Marketing Intelligence and Planning, vol. 8, $\mathrm{n}^{\circ}$ 7, Emerald Group Publishing Limited.

Perreault, J.D. (2004), Une nouvelle vision $d u$ service à la clientèle, document de travail, Laboratoire de recherche et d'intervention en gestion hôtelière et restauration, Département des sciences de la gestion, Université du Québec à Trois-Rivières, hiver.

Pettigrew, D., et N. Turgeon (2004), Le Marketing, $5^{\mathrm{e}}$ édition, Chenelière-McGraw-Hill.

Samii, A.K. (2001), Stratégies de service, Paris, Dunod, $314 \mathrm{p}$. 